\title{
SYMBOLIC METHODS FOR ANALYSING BIFURCATIONS AND CHAOS OF TWO FIVE-PARAMETER FAMILIES OF PLANAR QUADRATIC MAPS
}

\author{
Sarbast H. Mikaeel ${ }^{\text {a }}$, Bewar Beshayi ${ }^{\text {a }}$ \\ a Dept. of Mathematics, Faculty of Science, Soran University, Kurdistan Region, Iraq - (sarbast.mikael@soran.edu.iq; \\ bewar.osman@soran.edu.iq)
}

Received: Apr., 2020 / Accepted: Jun., 2020 / Published: Jun.,2020

https://doi.org/10.25271/sjuoz.2020.8.2.723

\begin{abstract}
:
In this work, we analyze the dynamical behaviors of two five-parameter families of planar quadratic maps by utilizing strategies of symbolic computation. We are going to use computer algebra methods to clarify how to detect the stability of equilibrium points to analyze chaos and also the bifurcation of planar maps. Based on strategies for solving the systems in types of semi-algebraic and by utilizing an algorithmic approach, we obtain respectively for the two maps, sufficient conditions on the parameters to have a prescribed number of (stable) equilibrium points; necessary conditions on the parameters to undergo a certain type of bifurcation or to have chaotic behavior induced by snapback repeller
\end{abstract}

KEYWORDS: Bifurcations, Chaos, Planar quadratic maps, Symbolic computation, Snapback repeller.

\section{INTRODUCTION}

The dynamical system has two different kinds, which are discrete and continuous, that are widely and typically used for the modeling of biological phenomena, control and economic problems (Hong et al., 2015; Kaslik \& Balint, 2009; Sang, \& Huang, 2017). Many of those kinds of systems are nonlinear with parameters, and generally their analytical solutions are complicate to find. For understanding the phenomena or the problems that involved in dynamical systems, it is important to investigate their behaviors like stability, bifurcations and chaos qualitatively.

Over the real field $\mathbb{R}$ for the discrete dynamical systems with state variables and parameters, for the discovery and investigation of stability and bifurcations of real equilibrium points, a common algebraic approach has been proposed in $(\mathrm{Li}$ et al., 2011). A short time ago, this approach, has moreover been applied to investigation of chaos actuated by snapback repeller for dynamical systems in discrete types (Huang \& Niu 2019a). In this study, we discuss the problem of analyzing the dynamical behaviors of planar quadratic discrete dynamical systems, which is proposed in (Huang \& Niu, 2020c).

Problem. Examine a planar quadratic map as follows:

$$
\begin{aligned}
x_{n+1}= & a_{1,1} x_{n}^{2}+a_{1,2} x_{n} y_{n}+a_{1,3} y_{n}^{2} \\
& +a_{1,4} x_{n}+a_{1,5} y_{n}+a_{1,6} \\
y_{n+1}= & a_{2,1} x_{n}^{2}+a_{2,2} x_{n} y_{n}+a_{2,3} y_{n}^{2} \\
& +a_{2,4} x_{n}+a_{2,5} y_{n}+a_{2,6} .
\end{aligned}
$$

We assume that $\bar{x}$ is an equilibrium point of the above system (1), (if it exists). To determine the conditions explicitly according the parameters $a_{i, j}$ for $i=1,2, j=1, \ldots, 6$, such that:

1. $\bar{x}$ is the stable equilibrium point for system (1);

2. The system (1) go through certain sorts of bifurcations;

3. And finally, in system (1), $\bar{x}$ is a snapback repeller. Our study is focus on the period doubling bifurcation (PDB) and the neimark-sacher bifurcation (NSB), for the discrete system (1). In recent years, those kind of bifurcations for discrete systems have been discussed by many researchers. We refer the interested readers to (Din, 2017; He \& Lai, 2011; Niu et al., 2016) for more details on bifurcation analysis. Comment that, numerous of well-studied and famous maps are subclasses of the system (1), like generalized Hénon map and Tinkerbell map (Alligood et al., 1996; Davidchack, 2001; Hénon, 1976) and generalized Mira 2 map (Huang \& Niu, 2020c; Alexandra et al., 1996). In this work we will pay particular attention to certain subclasses of system (1). More concretely, we consider the family

$$
\begin{gathered}
x_{n+1}=a x_{n}+b y_{n}, \\
y_{n+1}=c x_{n}^{2}+k x_{n} y_{n}+e y_{n}^{2},
\end{gathered}
$$

where the parameters $a, b, c, k, e$ are in $\mathbb{R}^{+}$with $a \neq 1$; and we consider the family

$$
\begin{aligned}
& x_{n+1}=E y_{n}^{2}+B y_{n}+K, \\
& y_{n+1}=C x_{n}^{2}+A x_{n}+K,
\end{aligned}
$$

where the parameters $A, B, C, K, E$ are in $\mathbb{R}^{+}$.

The aim of this paper is to study the conditions on the parameters for the planar maps (2) and (3) algebraically and symbolically, to have a number of prescribed (stable) equilibrium points, the NSB and PDB types, and chaos induced by snapback repeller. The main technique is to use an algorithmic algebraic approach based on the triangular decomposition (Wu, 2000; Wang, 2001), quantifier elimination (Collins \& Hong, 1991; Hong et al.,1997), Gröbner bases (Buchberger, 1985; Faugère, 2002), discriminant varieties (Lazard \& Rouillier, 2007), and real solution classification (Yang \& Xia, 2005). This paper is organized as follows. We show and explain how to diminish the stability, bifurcations and chaos problem of planar maps to simply arithmetical problems and after that fathom these problems by utilizing algebraic methods, in section 2 . The experimental results of the two parametric planar quadratic maps (2) and (3) are provided in section 3 together with some remarks. Finally, we have done this paper with a conclusion in the last section, section 4 .

* Corresponding author

This is an open access under a CC BY-NC-SA 4.0 license (https://creativecommons.org/licenses/by-nc-sa/4.0/) 


\section{ALGEBRAIC CRITERIA FOR STABILITY, BIFURCATIONS AND CHAOS OF PLANAR MAPS}

Consider the following 2-dimensional discrete dynamical systems

$$
x(t+1)=g(\mu, x(t))
$$

where $x(t+1), x(t) \in \mathbb{R}^{2}$, are the state vectors, and $g$ is a $\mathcal{C}^{1}$ non-linear map with parameters $\mu$, from the field of real number $\mathbb{R}$.

First, we are going to describe a few ideas for system (4). Assume $g^{k}$ denote the compositions of $g$ in $k$ times with itself. And if $g^{p}(x)=x$, a point $\mathrm{x}$, is said to be a $p$-periodic point of $g$ but $g^{k} \neq x$ for $\mathrm{p}>\mathrm{k} \geq 1$. And $x$ is called an equilibrium point if $p=1$, i.e., $g(x)=x$. Let the Jacobian matrix of $g$ is $g^{\prime}(x)$ with determinant $\left|g^{\prime}(x)\right|$. In the following subsections, we clarify how to decrease the problems of bifurcations, stability and chaos analyses of system (4) to algebraic problems.

\subsection{Stability Analysis of Equilibrium Points}

Observably, to determine the equilibrium points of system (4), we are able to use the equation: $[x-g(\mu, x)=0]$. We need to analyze the stability of (4) at each equilibrium point or to determine the conditions on the parameters for the equilibrium point to be stable(steady), after computing the equilibrium points. To this conclusion, we let:

$$
P(\lambda)=\lambda^{2}+a_{1} \lambda+a_{2}
$$

to the Jacobian matrix $g^{\prime}(x)$ of system (4) be the characteristic polynomial, where $a_{i}=a_{i}(\mu ; x), i=1,2$. For a discrete system in the form (4) to know how to determine the stability of an equilibrium point, we consider the following lemma.

Lemma 1. (Galor, 2007) Let $\bar{x}$ be a fixed point of system (4). If both roots of the polynomial (5) are inside the unit circle, then $\bar{x}$ is asymptotically stable.

To determine the arithmetic criterion for all zeros of a given polynomial to be outside the unit circle (OUC) or inside the unit circle (IUC). One may use a sequence of descending degrees of symmetric polynomials for the characteristic polynomial, look at (Bistritz, 1984; Huang \& Niu, 2019 a ) Which is denoted by $P\left(\lambda^{\star}\right)$ be the responded polynomial of $P(\lambda)$, namely,

$P\left(\lambda^{\star}\right)=\lambda^{2} P\left(\lambda^{-1}\right)=1+a_{1} \lambda+a_{2} \lambda^{2}$.

Using the polynomial $P(\lambda)$ and $P\left(\lambda^{\star}\right)$, we can construct a sequence of three polynomials:

$$
\begin{aligned}
& L_{2}(\lambda)=P(\lambda)+P^{\star}(\lambda) \\
& L_{1}(\lambda)=\frac{P(\lambda)-P^{\star}(\lambda)}{\lambda-1} \\
& L_{0}(\lambda)=\frac{1}{\lambda}\left[\delta(\lambda+1) L_{1}(\lambda)-L_{2}(\lambda)\right],
\end{aligned}
$$

where $\delta=\frac{L_{2}(0)}{L_{1}(0)}$. The normal conditions are requires for the recursion, $L_{k}(0) \neq 0$ for $k=0,1,2$. When a $L_{k}(0)=0$ happens the construction is interrupted, and it divided in two type which is IUC and OUC (Bistritz, 1984). The following result follows directly from theorem 4 in (Huang, \& Niu, 2020c), or theorem 2 in (Huang \& Niu, 2019 b). The proofs of it can be found in (Bistritz, 1984; Huang \& Niu, 2019a).
Corollary 1. The normal conditions $L_{2-i} \neq 0, i=0,1,2$ hold and $v_{2}=\operatorname{Var}\left\{L_{2}(1), L_{1}(1), L_{0}(1)\right\}=0$ or $v_{2}=2$ if and only if all zeros of $P(\lambda)$ are IUC or OUC.

The notation $v_{2}$ denotes the number of symptom changes of the sequence of real numbers $\left\{L_{2}(1), L_{1}(1), L_{0}(1)\right\}$. The result of OUC will be used in section 2.3 to derive algebraic criteria for the chaos analysis of system (4), so we present it here for brevity.

\subsection{Bifurcation Analysis}

When a particular parameter go through its critical value in dynamical systems, different sorts of bifurcations rise from its equilibrium point. Usually when the stability of an equilibrium point changes, the bifurcation occurs, i.e., when a dynamical system qualitative properties change. In this paper we look into the parametric conditions for the existence of the PDB and NSB, for the discrete system (4).

1. NSB. For this bifurcation, the Jacobian matrix $g^{\prime}(\bar{x})$ has a pair of complex conjugate eigenvalues on the unit cycle and all other eigenvalues (if they exist) interior the circle. A characteristic polynomial (5) of the Jacobian matrix $g^{\prime}(\bar{x})$ is given, we consider the following determinants $(\mathrm{Li}$ et al, 2011; Wen, 2005):

$$
\begin{aligned}
\Delta_{1}^{ \pm}(\mu, \bar{x}) & =\left|1 \pm a_{2}\right| \\
\Delta_{2}^{ \pm}(\mu, \bar{x}) & =\left|\left(\begin{array}{cc}
1 & a_{1} \\
0 & 1
\end{array}\right) \pm\left(\begin{array}{cc}
a_{1} & a_{2} \\
a_{2} & 0
\end{array}\right)\right| .
\end{aligned}
$$

Then, system (4) may undergo a NSB if the following conditions hold:

(a) $P(1)>0$ and $P(-1)>0$,

(b) $\Delta_{1}^{-}(\mu, \bar{x})=0, \Delta_{1}^{+}(\mu, \bar{x})>0, \Delta_{2}^{ \pm}(\mu, \bar{x})>0$,

2. PDB. A PDB (also called flip bifurcation) which can only arise in a discrete dynamical system. The system switches to an unused behavior with twice the period of the original system, at this bifurcation. In this situation, when a series of PDBs may lead the system from order to chaos. The Jacobian matrix $g^{\prime}(\bar{x})$ has one real eigenvalue which is equal to -1 , and the remain of eigenvalues of $g^{\prime}(\bar{x})$ are all in the interior of the unit circle. Obviously, system (4) may undergo a PDB if the following conditions hold:

a) $\mathrm{P}(-1)=0$ and $\mathrm{P}(1)>0$,

b) $\Delta_{1}^{ \pm}(\mu, \bar{x})>0, \Delta_{2}^{ \pm}(\mu, \bar{x})>0$.

We notice that the above conditions for the bifurcations analyzed are also become to the critical conditions for an eigenvalue to reach the unit circle. This eigenvalue ought to not be stationary on the unit circle, but proceed to go exterior of the circle as the parameters change. Furthermore, the conditions of critical with the so-called transversality and the non-resonance decide whether a bifurcation can be produced or not. In this paper, we center our consider on the critical conditions, which gives conceivable outcomes for the occurrence of bifurcations. The induction of necessary and sufficient bifurcation conditions and further analysis of the kinds and stability of bifurcations are our ongoing research.

\subsection{Marotto's Theorem}

Let us describe the notion of Marotto's theorem and snapback repeller. Consider a $\mathcal{C}^{1}$ non-linear map (4). Let $B_{r}(x)$ be a closed ball defined on norm $\|\cdot\|$ in $\mathbb{R}^{2}$, where $a$ is radius and $x \in \mathbb{R}^{2}$ is the center of the closed ball. An equilibrium point $\bar{x}$ 
is repelling equilibrium point of $g$ according to the norm $\|$.$\| if$ there exists $c>1$ (where $c$ is constant), such that $\| g(x)-$ $g(y)\|>c\| x-$.$y \| for any x, y \in B_{r}(\bar{x})$ with $x \neq y$, where $B_{r}(\bar{x})$ is called a repelling neighborhood of $\bar{x}$.

We say that a repelling equilibrium point $\bar{x}$ is called a snapback repeller of $g$ if there exist a point $x_{0} \in B_{r}(\bar{x})$ with $x_{0} \neq$ $\bar{x}$ and a positive integer $s>1$, such that $x_{s}=\bar{x}$ and $\left|g^{\prime}\left(x_{k}\right)\right| \neq 0$ for $1 \leq k \leq \mathrm{s}$, where $x_{k}=g^{k}\left(x_{0}\right)$. Moreover, the point $x_{0}$ is called a snapback point of $g$. Then, the following theorem according to Marotto is hold (Marotto, 2005; Marotto, \& FR, 1987)

Theorem 1. If $g$ possesses a snapback repeller, then $g$ is chaotic in the sense of Marotto.

By using the following lemma from (Galor, 2007) which can be used to the repelling equilibrium point of $g$ that can be determined under the Euclidean norm.

Lemma 2. Let $\bar{x}$ be an equilibrium point of $g$ which is continuously differentiable in $B_{r}(\bar{x})$. If

$$
\lambda>1 \text {, for all eigenvalues } \lambda \text { of }\left(g^{\prime}(\bar{x})\right)^{T} g^{\prime}(\bar{x}) \text {, }
$$

then there exist $s>1$ and $r^{\prime} \in(0, r]$ such that $\| g(x)-$ $g(y)\left\|_{2}>s.\right\| x-y \|_{2}$ for all $x, y \in B_{r}(\bar{x})$ with $x \neq y$, and all the eigenvalues of $\left(g^{\prime}(x)\right)^{T} g^{\prime}(x)$ exceed one for all $x \in$ $B_{r^{\prime}}(\bar{x})$.

We have clarified how to formulate a critical algebraic criterion for all zeros of a given polynomial to be OUC (see Corollary 1). According to this criterion, we can summarize the critical algebraic conditions for analyzing the Marotto's theorem. Let $\bar{P}(\lambda)=\lambda^{2}+\bar{a}_{1} \lambda+\bar{a}_{2}$ be the characteristic polynomial of $\left(g^{\prime}(\bar{x})\right)^{T} g^{\prime}(\bar{x})$ where $\bar{a}_{i}=\bar{a}_{i}(\mu, \bar{x}), \quad i=1,2$.

Due to equation (6) we can relate with $P(\lambda)$ a sequence $\left\{\bar{L}_{0}(\lambda), \bar{L}_{1}(\lambda), \bar{L}_{2}(\lambda)\right\}$. More accurately, the following theorem is used to analyze the chaotic behavior of system (4).

Theorem 2. (Huang \& Niu 2019a; Huang \& Niu, 2019 b) For a planar discrete system (4), the system is chaotic in the sense of Marotto if one of the following semi-algebraic systems have at least one real solution:

$$
\psi_{j}:\left\{\begin{array}{c}
\bar{x}-g(\mu, \bar{x})=0, \quad g^{s}\left(x_{0}\right)-\bar{x}=0 \\
\left.(-1)^{i+j-1} \bar{L}_{2-i}(1)\right|_{\bar{x}}>0, i=1,2 \\
\left.(-1)^{i+j-1} \bar{L}_{2-i}(1)\right|_{x_{0}}>0, i=1,2, \\
\left.\bar{L}_{2-i}(0)\right|_{x_{0}} \neq 0,\left.\bar{L}_{2-i}(0)\right|_{\bar{x}} \neq 0, i=0,1,2 \\
x_{0} \neq \bar{x},\left|g^{\prime}\left(x_{k}\right)\right| \neq 0, k=1, \ldots, s
\end{array}\right.
$$

$j=1,2, \mu$ and $\bar{x}$ are respectively the parameters and equilibrium point of system (4) and $s \geq 2$ is a given positive integer number.

Remark 1. Note that as the authors stated in (Huang \& Niu 2019a) that the property of the sign variety design in Lemma 4.1 in (Huang \& Niu 2019a) can offer assistance us to discover a repelling neighborhood $B_{r^{\prime}}(\bar{x})$ (where $r^{\prime}$ is sufficiently small), but the radius $r^{\prime}$ may not be adequately little sufficient to make $B_{r^{\prime}}(\bar{x})$ be a thorough repelling neighborhood of the equilibrium point $\bar{x}$. That is to say Theorem 2 gives a probabilistic criterion for a planar discrete system (4) to have a snapback repeller. How to derive critical algebraic criteria for finding a rigorous repelling neighborhood of the equilibrium point $\bar{x}$ is a question for further study.

Our goal is to find the parametric conditions on the parameters $\mu$ for each of the semi-algebraic system (9) to have at least one real solution. There exist algebraic methods based on Gröbner bases, triangular decomposition, quantifier elimination, real solution classification, and discriminant varieties which can be used to solve such semi-algebraic systems. In next section, we discuss the performance of the algebraic methods by analysing the dynamical behaviors of the parametric systems (2) and (3).

\section{EXPERIMENTAL RESULTS}

In this section, we focus to investigate the stability, chaos and bifurcations for systems (2) and (3). All the calculations were made by Maple 17, running under windows 8 professional edition on an Intel(R) Core(TM)i7-7500U CPU @ 1.9GHz 2.49Ghz with $8 \mathrm{G}$ RAM.

\subsection{Analysis of Bifurcations and Chaos for System (2)}

First we detect the equilibrium points of system (2) by using the algebraic system as follows:

$$
\left\{\begin{array}{c}
P_{1}=a x+b y-x=0, \\
P_{2}=c x^{2}+k x y+c y^{2}-y=0 .
\end{array}\right.
$$

Where $a, b, c, k, e$ are positive real number with $a-1 \neq 0$. Notice that the Jacobian matrix $g^{\prime}(\bar{x})$ of map (2) assessed at the equilibrium point $\bar{x}=(x, y)$ is given by $g^{\prime}(\bar{x})=$ $\left(\begin{array}{cc}a & b \\ 2 c x+k y & 2 e y+k x\end{array}\right)$, and also the characteristic polynomial of the matrix $g^{\prime}(\bar{x})$ can be written as

$$
\begin{aligned}
& P(\lambda)=\lambda^{2}+(-2 e y-k x-a) \lambda+2 a e y+a k x \\
& \quad-2 b c x-b k y .
\end{aligned}
$$

To evaluate the stability of each equilibrium point, we first use Corollary 1 together with equation (6) for the polynomial $P(\lambda)$ to obtain inequality polynomials $L_{2}(1), L_{1}(1), L_{0}(1)$ and $L_{2}(0), L_{1}(0), L_{0}(0)$ and then reduce the problem to that of solving the following semi-algebraic system

$$
\left\{\begin{array}{c}
P_{1}=a x+b y-x=0, \quad P_{2}=c x^{2}+k x y+e y^{2}-y=0, \\
L_{2}(1)=4 a e y+2 a k x-4 b c x-2 b k y-4 e y-2 k x \\
\quad-2 a+2>0, \quad\left(o r-L_{2}(1)>0\right), \\
\begin{array}{rl}
L_{1}(1)= & -4 a e y-2 a k x+4 b c x+2 b k y+2>0, \\
\left(o r-L_{1}(1)>0\right), & \\
L_{0}(1)= & 4 a e y+2 a k x-4 b c x-2 b k y+4 e y+2 k x \\
& +2 a+2>0,\left(o r-L_{0}(1)>0\right), \\
L_{2}(0)= & 2 a e y+a k x-2 b c x-b k y+1 \neq 0, \\
L_{1}(0)= & -2 a e y-a k x+2 b c x+b k y+1 \neq 0, \\
L_{0}(0)= & 4 a e y+2 a k x-4 b c x-2 b k y+4 e y+2 k x \\
& +2 a+2 \neq 0 .
\end{array}
\end{array}\right.
$$

Where $a, b, c, k, e$ are positive real number with $a-1 \neq 0$. By using the method of discriminant varieties of (Lazard \& Rouillier, 2007) (implemented as a Maple package DV by Moroz and Rouillier), or the method of (Yang \& Xia, 2005) for real solution classification (implemented as a Maple package DISCOVERER by Xia), the above semi-algebraic systems (10) and (11) may be solved. Firstly, we list the results on the classification for the number of (stable) equilibrium points.

(I) System (2) always has two (distinct) equilibrium points when $\left[a^{2} e-a b k+b^{2} c-\right.$ $2 a e+b k+e \neq 0]$.

(II) When one of the following conditions holds, system (2) has one stable equilibrium point.

$$
\begin{gathered}
A_{1}=\left[R_{1,1}<0,0<R_{1,2}, R_{1,4}<0\right], \\
A_{2}=\left[R_{1,1}<0,0<R_{1,2}, 0<R_{1,3}, 0<R_{1,4}\right], \\
A_{3}=\left[0<R_{1,1}, R_{1,2}<0, R_{1,3}<0,0<R_{1,4}\right], \\
A_{4}=\left[0<R_{1,1}, 0<R_{1,2}, 0<R_{1,3}, R_{1,4}<0\right],
\end{gathered}
$$$$
\text { where }
$$ 


$$
R_{1,1}=a-1 \text {, }
$$

$R_{1,2}=a^{2} e-a b k+b^{2} c-2 a e+b k+e$,

$R_{1,3}=3 a^{3} e-3 a^{2} b k+3 a b^{2} c-3 a^{2} e+2 a b k$ $-b^{2} c-3 a e+b k+3 e$,

$R_{1,4}=2 a^{3} e-2 a^{2} b k+2 a b^{2} c-5 a^{2} e+4 a b k$ $-3 b^{2} c+4 a e-2 b k-e$.

System (2) cannot have two stable equilibrium points.

Next we determine the fundamental bifurcation conditions on parameters $a, b, c, k, e$ for system (2) to have a certain sort of bifurcations. For the NSB, the problem may be formulated as that of recognizing the conditions for the taking after semialgebraic system to have at slightest one real solution:

$$
\left\{\begin{array}{l}
P_{1}=a x+b y-x=0 \\
P_{2}=c x^{2}+k x y+e y^{2}-y=0 \\
1-(2 e y+k x) a+2 b c x+b k y=0 \\
1+(2 e y+k x) a-2 b c x-b k y>0 \\
1-2 e y-k x-a+(2 e y+k x) a-2 b c x-b k y>0 \\
1+2 e y+k x+a+(2 e y+k x) a-2 b c x-b k y>0 .
\end{array}\right.
$$

Where $a, b, c, k, e$ are positive real number with $a-1 \neq 0$. After solving the above system, we discover that system (2) may undergo a NSB if one of the thirty-five conditions holds:

$$
\begin{aligned}
& B_{1}=\left[R_{1,5}<0, R_{1,6} \leq 0,0<R_{1,7}, R_{1,8} \leq 0,\right. \\
& 0<R_{1,9}, R_{1,10} \leq 0, R_{1,11}, \leq 0,0 \leq R_{1,12}, \\
& 0 \leq R_{1,13}, R_{1,14}<0,0 \leq R_{1,16}, 0 \leq R_{1,17} \\
& \text {, } \left.S_{1}=0\right] \text {, } \\
& B_{2}=\left[R_{1,5}<0, R_{1,6} \leq 0,0<R_{1,7}, R_{1,8} \leq 0,\right. \\
& 0<R_{1,9}, R_{1,10} \leq 0, R_{1,11} \leq 0, R_{1,13} \leq 0 \text {, } \\
& \left.0<R_{1,14}, 0 \leq R_{1,15}, R_{1,17} \leq 0, S_{1}=0\right] \text {, } \\
& B_{35}=\left[0 \leq R_{1,6}, 0<R_{1,7}, 0 \leq R_{1,8}, R_{1,10} \leq 0\right. \text {, } \\
& 0 \leq R_{1,11}, 0 \leq R_{1,12}, 0 \leq R_{1,13}, R_{1.14}<0 \text {, } \\
& \text {, } \left.R_{1,15} \leq 0,, 0 \leq R_{1,16}, S_{1}=0\right] \text {. }
\end{aligned}
$$

where

$$
\begin{gathered}
R_{1,5}=4 c e-k^{2} \\
R_{1,6}=4 b^{2} c-4 b k-e \\
R_{1,7}=3 b^{2} c+2 b k+e \\
R_{1,8}=12 b^{2} c-10 b k-e \\
R_{1,9}=7 b^{2} c-32 b k+144 e \\
R_{1,10}=12 b^{2} c e-4 b^{2} k^{2}+4 b e k-e^{2} \\
R_{1,11}=16 b^{4} c^{2}-24 b^{3} c k+16 b^{2} c e+2 b e k-e^{2} \\
R_{1,12}=112 b^{4} c^{2}-232 b^{3} c k+176 b^{2} c e+64 b^{2} k^{2} \\
\quad-2 b e k+9 e^{2} \\
R_{1,13}=16 b^{3} c^{2} e-4 b^{3} c k^{2}-12 b^{2} c e k+4 b^{2} k^{3} \\
\quad-8 b c e^{2}-4 b e k^{2}+e^{2} k \\
R_{1,14}=16 b^{4} c^{2} e-4 b^{4} c k^{2}-32 b^{3} c e k+8 b^{3} k^{3} \\
\quad+44 b^{2} c e^{2}-12 b^{2} e k^{2}+6 b e^{2} k-e^{3} \\
R_{1,15}=16 b^{4} c^{2} e k-4 b^{4} c k^{3}-48 b^{3} c^{2} e^{2} \\
\quad-12 b^{3} c e k^{2}+6 b^{3} k^{4}+60 b^{2} c e^{2} k-17 b^{2} e k^{3} \\
+4 b c e^{2}+11 b e^{2} k^{2}-2 e^{3} k \\
R_{1,16}=48 b^{4} c^{2} e k-12 b^{4} c k^{3}-80 b^{3} c^{2} e^{2} \\
\quad-6 b^{3} c e k^{2}+8 b^{3} k^{4}-16 b^{2} c e^{2} k-4 b^{2} e k^{3} \\
-10 b c e^{3}-2 b e^{2} k^{2}+e^{3} k \\
R_{1,17}=112 b^{5} c^{3} e k-28 b^{5} c^{2} k^{3}+18 e^{3} k^{2} \\
\quad-1232 b^{4} c^{3} e^{2}+76 b^{4} c^{2} e k^{2}+58 b^{4} c k^{4} \\
\quad+2532 b^{3} c^{2} e^{2} k-583 b^{3} c e k^{3}-16 b^{3} k^{5} \\
\quad-1828 b^{2} c^{2} e^{3}+333 b^{2} c e^{2} k^{2}+88 b^{2} e k^{4} \\
-574 b c e^{3} k-76 b e^{2} k^{3}+72 c e^{4} \\
S_{1}=2 a^{3} e-2 a^{2} b k+2 a b^{2} c-5 a^{2} e+4 a b k \\
-3 b^{2} c+4 a e-2 b k-e
\end{gathered}
$$

Similarly, we also find that system (2) may experience (undergo) a PDB if one of the twenty-four conditions holds:

$$
\begin{gathered}
C_{1}=\left[R_{1,18}<0,0<R_{1,19}, R_{1,20}<0, R_{1,21} \leq 0\right. \\
R_{1,22} \leq 0, R_{1,23}<0, R_{1,24} \leq 0, R_{1,25} \leq 0 \\
0 \leq R_{1,27}, R_{1,28}<0,0 \leq R_{1,29}, 0 \leq R_{1,30} \\
\left.0 \leq R_{1,31}, S_{2}=0\right] \\
C_{2}=\left[R_{1,18}<0,0<R_{1,19}, R_{1,20}<0, R_{1,21} \leq 0\right. \\
R_{1,22} \leq 0, R_{1,24} \leq 0, R_{1,25} \leq 0,0 \leq R_{1,26} \\
0 \leq R_{1,27}, R_{1,28}<0,0 \leq R_{1,29}, 0 \leq R_{1,30} \\
\left.R_{1,31} \leq 0, S_{2}=0,\right] \\
\quad \begin{array}{c}
C_{24}=\left[0<R_{1,19}, R_{1,20}<0,0 \leq R_{1,22}, 0<R_{1,23}\right. \\
R_{1,24} \leq 0,0 \leq R_{1,25}, 0 \leq R_{1,26}, 0 \leq R_{1,27} \\
R_{1,28}<0, R_{1,29} \leq 0,0 \leq R_{1,30}, R_{1,31} \leq 0
\end{array} \\
\left.S_{2}=0\right]
\end{gathered}
$$

Where

$$
\begin{aligned}
& R_{1,18}=c e-1 \text {, } \\
& R_{1,19}=b c+2 \text {, } \\
& R_{1,20}=b^{2} c-2 b-3 e \text {. } \\
& R_{1,21}=3 b c-2 \text {, } \\
& R_{1,22}=3 b c e-4 b+6 e \text {, } \\
& R_{1,23}=7 b^{2} c-64 b+144 e \text {, } \\
& R_{1,24}=3 b^{2} c e-4 b^{2}-4 e^{2} \text {, } \\
& R_{1,25}=3 b^{2} c^{2} e+4 b c-4 b+4 e \text {, } \\
& R_{1,26}=21 b^{2} c^{2} e-20 b c e-48 c e^{2}-4 b \\
& +92 e \text {, } \\
& R_{1,27}=9 b^{3} c^{2} e+9 b^{2} c^{2} e^{2}-9 b^{3} c-18 b^{2} c e+ \\
& 12 b c e^{2}+12 b^{2}-16 b e+12 e^{2}, \\
& R_{1,28}=27 b^{4} c^{3} e-27 b^{4} c^{2}-108 b^{2} c^{2} e^{2} \\
& +288 b^{2} c e+144 b c e^{2}+144 c e^{3} \\
& -192 b^{2}-128 b e-192 e^{2} \\
& R_{1,29}=27 b^{4} c^{3} e^{2}-27 b^{4} c^{2} e+162 b^{3} c^{2} e^{2} \\
& -18 b^{2} c^{2} e^{3}-396 b^{3} c e+48 b^{2} c e^{2} \\
& -144 b c e^{3}+240 b^{3}-56 b^{2} e \\
& +192 b e^{2}-72 e^{3} \text {, } \\
& R_{1,30}=81 b^{4} c^{4} e^{2}+54 b^{3} c^{4} e^{3}-189 b^{4} c^{3} e \\
& -90 b^{3} c^{3} e^{2}+72 b^{2} c^{3} e^{3}+108 b^{4} c^{2} \\
& +72 b^{3} c^{2} e-156 b^{2} c^{2} e^{2}+72 b c^{2} e^{3} \\
& -36 b^{3} c+12 b^{2} c e-168 b c e^{2}+96 b^{2} \\
& +64 b e+96 e^{2} \\
& R_{1,31}=567 b^{5} c^{4} e^{2}-594 b^{4} c^{4} e^{3}-1323 b^{5} c^{3} e \\
& +1638 b^{4} c^{3} e^{2}-360 b^{3} c^{3} e^{3}+3024 b^{2} c^{3} e^{4} \\
& +756 b^{5} c^{2}-144 b^{4} c^{2} e+1500 b^{3} c^{2} e^{2} \\
& -9864 b^{2} c^{2} e^{3}+5184 b c^{2} e^{4}-900 b^{4} c \\
& -3948 b^{3} c e+14136 b^{2} c e^{2}-9216 b c e^{3} \\
& +5184 c e^{4}+2976 b^{3}-8384 b^{2} e+5856 b e^{2} \\
& -8640 e^{3} \text {, } \\
& S_{2}=3 a^{3} e+3 a b^{2} c-6 a^{2} b-3 a^{2} e-b^{2} c+ \\
& 4 a b-3 a e+2 b+3 e \text {, }
\end{aligned}
$$

Remark that, the above necessary conditions for the PDB is obtained when $k=2$. Since Maple was expending as well much of the CPU amid the calculation for the five free parameters $a, b, c, k, e$.

Finally, we are going to determine the conditions on $a, b, c, k, e$ under which the equilibrium point $\bar{x}$ of system (2) may be a snapback repeller. According to Marotto's theorem, we ought to discover one point $x_{0}=\left(x_{0}, y_{0}\right)$ in a repelling neighbourhood $B_{r^{\prime}}(\bar{x})$ such that $x_{0} \neq \bar{x}, g^{S}\left(x_{0}\right)=\bar{x}$ and $\left|g^{\prime}\left(x_{k}\right)\right| \neq 0,(1 \leq k \leq s)$ for some positive integer $m$. Here, we particularly focus on the map $g^{2}(m=2)$. Note that the characteristic polynomial of the matrix $\left(g^{\prime}(\bar{x})\right)^{T} g^{\prime}(\bar{x})$ is

$$
\bar{P}(\lambda)=\lambda^{2}+\left(-4 c^{2} x^{2}-4 c k x y-4 e^{2} y^{2}\right.
$$




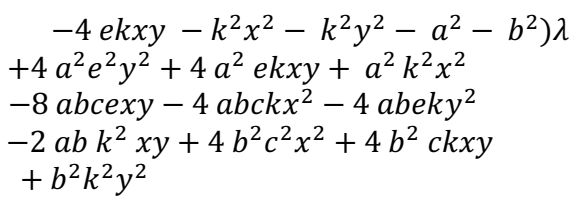

For the above polynomial $\bar{P}(\lambda)$ we can obtain the inequality polynomials $\bar{L}_{2}(1), \bar{L}_{1}(1), \bar{L}_{0}(1)$ and $\bar{L}_{2}(0), \bar{L}_{1}(0), \bar{L}_{0}(0)$ in Theorem 2 by using equation (6).

In arrange to analyse the chaotic behaviour of system (2), by Theorem 2 we obtain the following semi-algebraic system for $j=1$ :

$$
\begin{aligned}
& \left(P_{1}=a x+b y-x=0, P_{2}=c x^{2}+k x y+e y^{2}-y=0,\right. \\
& P_{3}=a\left(a x_{0}+b y_{0}\right)+b\left(c x_{0}^{2}+k x_{0} y_{0}+e y_{0}^{2}\right)-x=0 \text {, } \\
& P_{4}=c\left(a x_{0}+b y_{0}\right)^{2}+k\left(a x_{0}+b y_{0}\right)\left(c x_{0}^{2}+k x_{0} y_{0}\right. \\
& \left.+e y_{0}^{2}\right)+e\left(c x_{0}^{2}+k x_{0} y_{0}+e y_{0}^{2}\right)^{2}-y=0, \\
& \psi_{1}:\left\{\begin{array}{l}
\left.\bar{L}_{2}(1)\right|_{\bar{x}, x_{0}}>0, \quad-\left.\bar{L}_{1}(1)\right|_{\bar{x}, x_{0}}>0,\left.\bar{L}_{0}(1)\right|_{\bar{x}, x_{0}}>0, \\
\left.\bar{L}_{2}(0)\right|_{\bar{x}, x_{0}} \neq 0,\left.\bar{L}_{1}(0)\right|_{\bar{x}_{,} x_{0}} \neq 0,\left.\bar{L}_{0}(0)\right|_{\bar{x}, x_{0}} \neq 0,
\end{array}\right. \\
& \left|g^{\prime}\left(x_{1}\right)\right|=(2 a e-b k)\left(c x_{0}{ }^{2}+k x_{0} y_{0}+e y_{0}{ }^{2}\right) \\
& +(a k-2 b c)\left(a x_{0}+b y_{0}\right) \neq 0 \text {, } \\
& \begin{array}{c}
\left|g^{\prime}\left(x_{2}\right)\right|=(2 a e-b k) y+(a k-2 b c) x \neq 0, \\
x_{0}-\bar{x} \neq 0 .
\end{array}
\end{aligned}
$$

Where $a, b, c, k, e$ are positive real number with $a-1 \neq 0$, and

$$
\begin{gathered}
\left.\bar{L}_{2}(1)\right|_{\bar{x}}=8 a^{2} e^{2} y^{2}+8 a^{2} e k x y+2 a^{2} k^{2} x^{2}-2 b^{2} \\
-16 a b c e x y-8 a b c k x^{2}-8 a b e k y^{2}-4 a b k^{2} x y \\
+8 b^{2} c^{2} x^{2}+8 b^{2} c k x y+2 b^{2} k^{2} y^{2}-8 c^{2} x^{2}+2 \\
-8 c k x y-8 e^{2} y^{2}-8 e k x y-2 k^{2} x^{2}-2 k^{2} y^{2}
\end{gathered}
$$$$
-2 a^{2}
$$

$\left.\bar{L}_{1}(1)\right|_{\bar{x}}=-8 a^{2} e^{2} y^{2}-8 a^{2} e k x y-2 a^{2} k^{2} x^{2}+2$ $+16 a b c e x y+8 a b c k x^{2}+8 a b e k y^{2}$ $-2 b^{2} k^{2} y^{2}$ $+4 a b k^{2} x y-8 b^{2} c^{2} x^{2}-8 b^{2} c k x y$

$\left.\bar{L}_{0}(1)\right|_{\bar{x}}=8 a^{2} e^{2} y^{2}+8 a^{2} e k x y+2 a^{2} k^{2} x^{2}+2 b^{2}$ $-16 a b c e x y-8 a b c k x^{2}-8 a b e k y^{2}+2 a^{2}$ $+8 b^{2} c^{2} x^{2}+8 b^{2} c k x y+2 b^{2} k^{2} y^{2}+8 c^{2} x^{2}$ $+8 c k x y+8 e^{2} y^{2}+8 e k x y+2 k^{2} x^{2}+2 k^{2} y^{2}$ $+2-4 a b k^{2} x y$,

$\left.\bar{L}_{2}(0)\right|_{\bar{x}}=4 a^{2} e^{2} y^{2}+4 a^{2} e k x y+a^{2} k^{2} x^{2}$ $-8 a b c e x y-4 a b c k x^{2}-4 a b e k y^{2}$ $+4 b^{2} c^{2} x^{2}+4 b^{2} c k x y-2 a b k^{2} x y$ $+b^{2} k^{2} y^{2}+1$,

$\left.\bar{L}_{1}(0)\right|_{\bar{x}}=-4 a^{2} e^{2} y^{2}-4 a^{2} e k x y-a^{2} k^{2} x^{2}$ $+8 a b c e x y+4 a b c k x^{2}+4 a b e k y^{2}$ $+2 a b k^{2} x y-4 b^{2} c^{2} x^{2}-4 b^{2} c k x y$ $-b^{2} k^{2} y^{2}+1$

$$
\left.\bar{L}_{0}(0)\right|_{\bar{x}}=\left.\bar{L}_{0}(1)\right|_{\bar{x}}
$$

With $a, b, c, k, e$ are positive real parameters and $x, y, x_{0}, y_{0}$ are the variables.

The semi-algebraic system $\psi_{1}$ can be solved by using DISCOVERER, the resulting output shows that the system $\psi_{1}$ when $k=2, b=e=1$ has at least one real solution if and only if one of the following conditions holds:

$$
\begin{aligned}
& D_{1}=\left[0<R_{1,32}, 0<R_{1,33}\right], \\
& D_{2}=\left[0<R_{1,32}, R_{1,34}<0\right],
\end{aligned}
$$

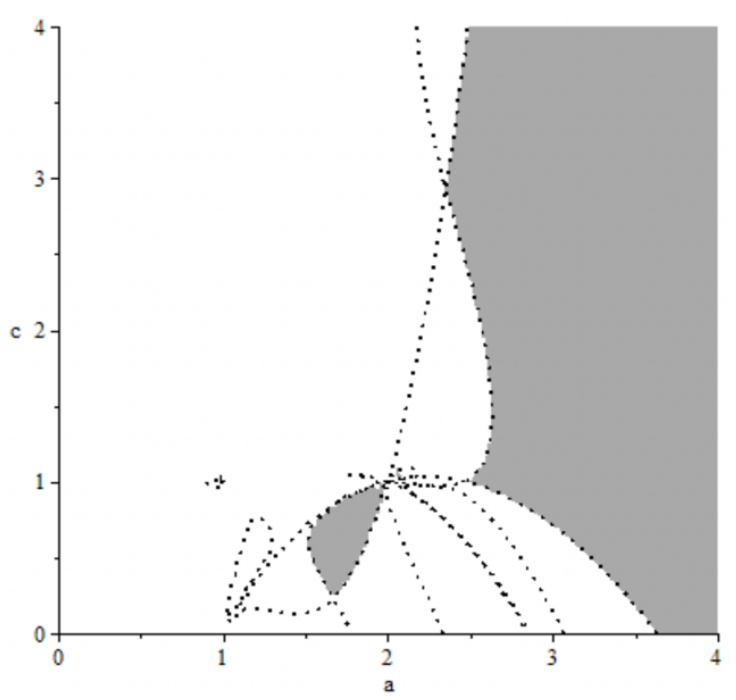

Figure 1. Parameter space for system (2) to be chaotic within the sense of Marroto.

Where

$$
\begin{aligned}
& R_{1,32}=3 a^{6}-24 a^{5}+6 a^{4} c+66 a^{4}-24 a^{3} c \\
& -a^{2} c^{2}-72 a^{3}+34 a^{2} c+11 a^{2}-16 a c \\
& +32 a-16 \text {, } \\
& R_{1,33}=3 a^{10}-36 a^{9}+12 a^{8} c+174 a^{8} \\
& -108 a^{7} c+14 a^{6} c^{2}-432 a^{7}+366 a^{6} c \\
& -76 a^{5} c^{2}+4 a^{4} c^{3}+579 a^{6}-608 a^{5} c \\
& +130 a^{4} c^{2}-4 a^{3} c^{3}-a^{2} c^{4}-364 a^{5} \\
& +574 a^{4} c-112 a^{3} c^{2}+2 a^{2} c^{3}-68 a^{4} \\
& -316 a^{3} c+59 a^{2} c^{2}+320 a^{3} \\
& +80 a^{2} c-16 a c^{2}-256 a^{2}+96 a-16, \\
& R_{1,34}=25 a^{24}-700 a^{23}+250 a^{22} c \\
& +8920 a^{22}-6220 a^{21} c+1085 a^{20} c^{2} \\
& -68400 a^{21}+69740 a^{20} c-23600 a^{19} c^{2} \\
& +351414 a^{20}-465328 a^{19} c+2680 a^{18} c^{3} \\
& +228448 a^{18} c^{2}-49904 a^{17} c^{3}+4146 a^{16} c^{4} \\
& -1272808 a^{19}+2053792 a^{18} c+3320276 a^{18} \\
& -1297232 a^{17} c^{2}+406688 a^{16} c^{3}+4156 a^{14} c^{5} \\
& -64264 a^{15} c^{4}-6295096 a^{17} c-51560 a^{13} c^{5} \\
& +4792736 a^{16} c^{2}-1906592 a^{15} c^{3}+425840 a^{14} c^{4} \\
& +2690 a^{12} c^{6}-6218208 a^{17}+13639140 a^{16} c \\
& -12060296 a^{15} c^{2}+5683056 a^{14} c^{3}-1577872 a^{13} c^{4} \\
& +264056 a^{12} c^{5}-25200 a^{11} c^{6}+1080 a^{10} c^{7} \\
& +8025417 a^{16}-20661808 a^{15} c+3588844 a^{12} c^{4} \\
& +20946004 a^{14} c^{2}-11221560 a^{13} c^{3}+245 a^{8} c^{8} \\
& -722848 a^{11} c^{5}+92064 a^{10} c^{6}-6960 a^{9} c^{7} \\
& -6097196 a^{15}+20523174 a^{14} c-24613744 a^{13} c^{2} \\
& +14766468 a^{12} c^{3}-5166040 a^{11} c^{4}+26 a^{6} c^{9} \\
& +1141312 a^{10} c^{5}-165424 a^{9} c^{6}+15744 a^{8} c^{7} \\
& +302300 a^{14}-9961596 a^{13} c+17839255 c^{2} \\
& +4647148 a^{10} c^{4}-1032680 a^{9} c^{5}-924 a^{7} c^{8} \\
& -12521456 a^{11} c^{3}+147056 a^{8} c^{6}+952 a^{6} c^{8} \\
& -14112 a^{7} c^{7}-44 a^{5} c^{9}+a^{4} c^{10}+5363760 a^{13} \\
& -4670704 a^{12} c-4665928 a^{11} c^{2}+6002292 a^{10} c^{3} \\
& -2436544 a^{9} c^{4}+476364 a^{8} c^{5}-45592 a^{7} c^{6} \\
& +1456 a^{6} c^{7}+64 a^{5} c^{8}-4 a^{4} c^{9}-6324880 a^{12} \\
& +12746656 a^{11} c-5119032 a^{10} c^{2}-612024 a^{9} c^{3} \\
& +627399 a^{8} c^{4}-66704 a^{7} c^{5}-16932 a^{6} c^{6} \\
& +4696 a^{5} c^{7}-434 a^{4} c^{8}+16 a^{3} c^{9}+2485296 a^{11} \\
& -10668224 a^{10} c+6983296 a^{9} c^{2}-1844 a^{4} c^{7} \\
& -1062224 a^{8} c^{3}-118524 a^{7} c^{4}+10118 a^{6} c^{5} \\
& +10128 a^{5} c^{6}+96 a^{3} c^{8}+1957456 a^{10} \\
& +3947904 a^{9} c-4314000 a^{8} c^{2}+719776 a^{7} c^{3} \\
& +152348 a^{6} c^{4}-46732 a^{5} c^{5}+3705 a^{4} c^{6}
\end{aligned}
$$




$$
\begin{aligned}
& -208 a^{3} c^{7}+16 a^{2} c^{8}-3474240 a^{9}+860032 a^{8} c \\
& +1676448 a^{7} c^{2}-319680 a^{6} c^{3}-108464 a^{5} c^{4} \\
& +31648 a^{4} c^{5}-2896 a^{3} c^{6}+2245280 a^{8} \\
& \quad-2049216 a^{7} c-439456 a^{6} c^{2}+182592 a^{5} c^{3} \\
& +128 a^{2} c^{7}+25344 a^{4} c^{4}-7744 a^{3} c^{5}+416 a^{2} c^{6} \\
& -499520 a^{7}+1357856 a^{6} c+59968 a^{5} c^{2} \\
& -97152 a^{4} c^{3}+2288 a^{3} c^{4}+608 a^{2} c^{5} \\
& -337664 a^{6}-532864 a^{5} c+16480 a^{4} c^{2} \\
& +32704 a^{3} c^{3}-1904 a^{2} c^{4}+349696 a^{5} \\
& +127360 a^{4} c-14144 a^{3} c^{2}-6176 a^{2} c^{3} \\
& +256 a c^{4}-1397764-14592 a^{3} c \\
& +4224 a^{2} c^{2}+512 a c^{3}+21760 a^{3}-512 a^{2} c \\
& -512 a c^{2}+3584 a^{2}+256 a c-2048 a \\
& +256 .
\end{aligned}
$$

In a few comparable steps, we will fathom and get that there's no given number of real solution(s) for the semi-algebraic system 2 when $k=2, b=e=1$. We notice that the polynomial expressions included within the analysis of chaotic behavior are huge, and Maple was unable to reclaim sufficient memory during a calculation indeed for three free parameters among $a, b, c, k, e$. In summary, we know that if one of the conditions: $D_{1}$ or $D_{2}$ is satisfied, the equilibrium point $\bar{x}$ of system (2) is a snapback repeller.

Now we take the conditions $D_{1} \cup D_{2}$ to demonstrate the parametric region where the equilibrium point $\bar{x}$ could be a snapback repeller for a visual understanding on the conditions above. The dotted lines in Figure 1 are the critical boundaries determined by the polynomials showing up in conditions $D_{1} \cup$ $D_{2}$, and the shadowed region is the parameter space where all the inequalities hold.

\subsection{Analysis of Bifurcations and Chaos for System (3)}

Since the calculations and arguments of bifurcations and chaos for system (3) are quite similar to those used in

the previous section, we omit the detailed steps. We summarize our results as follows.

(i) When $K=2, R_{2,1}>0$, system (3) has two (distinct) equilibrium points; when $K=$ $2, R_{2,1}<0 ; 0 \leq R_{2,2}, R_{2,3} \leq 0$, system (3) has four (distinct) equilibrium points.

(ii) When $K=2, E=B=1, R_{2,4}<0$, system (3) has two (distinct) equilibrium points, of which one is

stable; system (3) cannot have two or more stable equilibrium points.

(iii)

When $K=2, E=1, R_{2,5}=0$, system (3) may undergo a NSB, and no PDB occurs for this system.

(iv) When $A=10, B=5, C=2, E=1$ system (3) has a snapback repeller in case of the following conditions holds:

$$
\begin{gathered}
\varepsilon_{1}=[K>0,2 K-3<0], \\
\varepsilon_{2}=\left[0<2 K-3,4 K^{4}-52 K^{3}+227 K^{2}-375 K\right. \\
+203<0], \\
\varepsilon_{3}=\left[0<2 K-3,2 K^{2}-13 K+14<0\right] .
\end{gathered}
$$

The explicit expressions of $R_{2, i}, i=1,2, \ldots, 5$ are as follows:

$$
\begin{aligned}
& R_{2,1}=-A^{4} B^{4} C E+4 A^{5} B^{2} E^{2}+16 A^{2} B^{2} C E^{2}+ \\
& 4 A^{2} B^{5} C^{2}+16 A^{2} B^{4} C^{2} E-32 A^{5} E^{3}-64 A^{4} C E^{3}- \\
& 16 A^{3} B^{3} C E-64 A^{3} B^{2} C E^{2}-64 A^{2} B^{3} C^{2} E- \\
& 256 A^{2} B^{2} C^{2} E^{2}-32 B^{5} C^{3}-64 B^{4} C^{3} E-8 A^{4} B E^{2}- \\
& 32 A^{4} E^{3}+128 A^{3} B C E^{2}+512 A^{3} C E^{3}+256 A^{2} B C^{2} E^{2}+ \\
& 1024 A^{2} C^{2} E^{3}-8 A B^{4} C^{2}+128 A B^{3} C^{2} E+ \\
& 256 A B^{2} C^{2} E^{2}-32 B^{4} C^{3}+512 B^{3} C^{3} E+ \\
& 1024 B^{2} C^{3} E^{2}+62 A^{2} B^{2} C E+256 A^{2} B C E^{2}+ \\
& 512 A^{2} C E^{3}+256 A B^{2} C^{2} E-1024 A B C^{2} E^{2}- \\
& 2048 A C^{2} E^{3}+512 B^{2} C^{3} E-2048 B C^{3} E^{2}- \\
& 4096 C^{3} E^{3}+4 A^{3} E^{2}-240 A^{2} C E^{2}-1536 A C^{2} E^{2}+ \\
& 4 B^{3} C^{2}-240 B^{2} C^{2} E-1536 B C^{2} E^{2}-2048 C^{3} E^{2}- \\
& 2048 C^{2} E^{3}-72 A B C E-288 A C E^{2}-288 B C^{2} E- \\
& 1152 C^{2} E^{2}+27 C E \\
& R_{2,2}=A^{2} E-2 B C-8 C E \\
& R_{2,3}=-A^{2} B^{2} C E+2 A^{3} E^{2}+8 A^{2} C E^{2}+2 B^{3} C^{2} \\
& \quad+8 B^{2} C^{2} E-4 A B C E-16 A C E^{2}-16 B C^{2} E \\
& -64 C^{2} E^{2}+9 C E \\
& R_{2,4}=28 A^{5}+49 A^{4} C+40 A^{4}-560 A^{3} C- \\
& 980 A^{2} C^{2}-4 A^{3}-590 A^{2} C+3976 A C^{2}+6272 C^{3}+ \\
& +360 A C+5260 C^{2}-27 C \\
& R_{2,5}=-A^{4} B^{4} C+4 A^{2} B^{5} C^{2}+16 A^{2} B^{4} C^{2} \\
& \quad-32 B^{5} C^{3}+4 A^{5} B^{2}+16 A^{4} B^{2} C-16 A^{3} B^{3} C \\
& \quad-64 A^{2} B^{3} C^{2}-8 A B^{4} C^{2}-96 B^{4} C^{3}-64 A^{3} B^{2} C \\
& -256 A^{2} B^{2} C^{2}+128 A B^{3} C^{2}+512 B^{3} C^{3}-32 A^{5} \\
& \quad-8 A^{4} B-86 A^{4} C+128 A^{3} B C+70 A^{2} B^{2} C \\
& \quad+256 A^{2} B C^{2}+512 A B^{2} C^{2}-12 B^{3} C^{2} \\
& \quad+1536 B^{2} C^{3}-32 A^{4}+512 A^{3} C+256 A^{2} B C \\
& \quad+1024 A^{2} C^{2}-304 B^{2} C^{2}-2048 B C^{3}-12 A^{3} \\
& \quad+208 A^{2} C-40 A B C-3584 A C^{2}-1696 B C^{2} \\
& -6144 C^{3}-160 A C-2688 C^{2}-125 . \\
&
\end{aligned}
$$

We take the conditions $R_{2,4}<0$ and $R_{2,5}=0$ to illustrate the parametric region where system (3) has a stable equilibrium point and may have a NSB for a visual understanding. In summary, we provide in table 1 all the timings for analysing the dynamical behaviors of the parametric planar quadratic maps (2) and (3). 

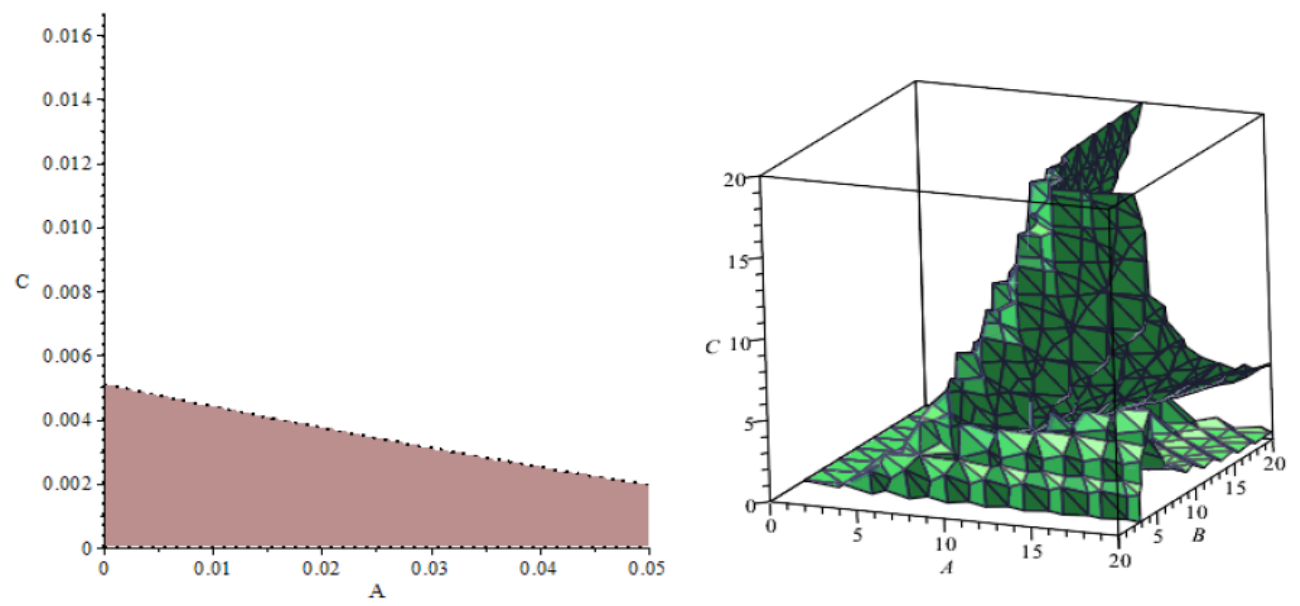

(a) System (3) has one stable equilibrium point.(b) Necessary conditions for system (3) to have a NSB.

Figure 2. Illustration of system (3).

Table 1. Runtimes (in seconds) on the obtained conditions for maps (2) and (3).

\begin{tabular}{|l|l|l|}
\hline Dynamical types & Map (2) & Map (2) \\
\hline Stability of equilibrium points & $0.844 \mathrm{~s}$ & $0.282 \mathrm{~s}$ \\
\hline NSB & $48.109 \mathrm{~s}$ & $1.047 \mathrm{~s}$ \\
\hline PDB & $33.187 \mathrm{~s}$ & -- \\
\hline Snapback repeller & $29.047 \mathrm{~s}$ & $11.406 \mathrm{~s}$ \\
\hline
\end{tabular}

\section{CONCLUSION}

Our experiments illustrate the viability and expediency of our algebraic approach for stability, bifurcation and chaos analysis of the planar quadratic maps (2) and (3). Due to the immensity of the polynomial expression in the analysis, computation has become very difficult to be done. As per some observations, it indicates conditions on the parameters for the bifurcation and chaos can be only attained under some restrictions. How to facilitate and enhance the steps of symbolic computations in the current approach stays a question which requires further study. The conditions for discrete dynamical system to attain a certain form of bifurcation or a snapback repeller we can obtain are only essential ones. The way of checking the adequacy of the conditions and determining the type of each bifurcation are areas worth of study. Furthermore, we are trying to prove an intriguing and challenging various models in different fields that stability, bifurcations and chaos could be significantly analyzed by our approach. The experimental results provide a partial answer to the problem stated in (Huang, \& Niu, 2020c).

\section{ACKNOWLEDGEMENTS}

The authors would like to thank Bo Huang for his helpful suggestions.

\section{REFERENCES}

Alligood, K. T., Sauer, T. D., \& Yorke, J. A. (1996). Chaos (pp. $105-$ 147). Springer New York.

Alexandra, B., Jean-claude, C., Laura, G., \& Christian, M. (1996). Chaotic dynamics in two-dimensional noninvertible maps (Vol. 20). World Scientific.

Bistritz, Y. U. V. A. L. (1984). Zero location with respect to the unit circle of discrete-time linear system polynomials. Proceedings of the IEEE, 72(9), 1131-1142.

Buchberger, B. (1985). Gröbner bases: An algorithmic method in polynomial ideal theory. Multidimensional systems theory.
Collins, G. E., \& Hong, H. (1991). Partial cylindrical algebraic decomposition for quantifier elimination. Journal of Symbolic Computation, 12(3), 299-328.

Davidchack, R. L., Lai, Y. C., Klebanoff, A., \& Bollt, E. M. (2001). Towards complete detection of unstable periodic orbits in chaotic systems. Physics Letters A, 287(1-2), 99-104.

Din, Q. (2017). Complexity and chaos control in a discrete-time preypredator model. Communications in Nonlinear Science and Numerical Simulation, 49, 113-134.

Faugère, J. C. (2002, July). A new efficient algorithm for computing Gröbner bases without reduction to zero (F 5). In Proceedings of the 2002 international symposium on Symbolic and algebraic computation (pp. 75-83).

Galor, O. (2007). Discrete dynamical systems. Springer Science \& Business Media.

He, Z., \& Lai, X. (2011). Bifurcation and chaotic behaviour of a discrete-time predator-prey system. Nonlinear Analysis: Real World Applications, 12(1), 403-417.

Hénon, M. (1976). A two-dimensional mapping with a strange attractor. In The Theory of Chaotic Attractors (pp. 94-102). Springer, New York, NY.

Hong, H., Liska, R., \& Steinberg, S. (1997). Testing stability by quantifier elimination. Journal of Symbolic Computation, 24(2), 161-187.

Hong, H., Tang, X., \& Xia, B. (2015). Special algorithm for stability analysis of multistable biological regulatory systems. Journal of Symbolic Computation, 70, 112-135.

Huang, B., \& Niu, W. (2019a). Analysis of snapback repellers using methods of symbolic computation. International Journal of Bifurcation and Chaos, 29(04), 1950054.

Huang, B., \& Niu, W. (2019b). Algebraic approach to chaos induced by snapback repeller. ACM Communications in Computer Algebra, 53(3), 122-125.

Huang, B., \& Niu, W. (2020c). Algebraic Analysis of Bifurcations and Chaos for Discrete Dynamical Systems. In International Conference on Mathematical Aspects of Computer and Information Sciences. MACIS 2019. Lecture Notes in Computer Science, pp. 169-184. Springer Nature.

Kaslik, E., \& Balint, S. (2009). Complex and chaotic dynamics in a discrete-time-delayed Hopfield neural network with ring architecture. Neural Networks, 22(10), 1411-1418.

Lazard, D., \& Rouillier, F. (2007). Solving parametric polynomial systems. Journal of Symbolic Computation, 42(6), 636667.

Li, C., \& Chen, G. (2003). An improved version of the Marotto theorem. Chaos, Solitons \& Fractals, 18(1), 69-77.

Li, X., Mou, C., Niu, W., \& Wang, D. (2011). Stability analysis for discrete biological models using algebraic methods. Mathematics in Computer Science, 5(3), 247262.

Marotto, F. R., \& FR, M. (1978). Snap-Back Repellers Imply Chaos In RN.

Marotto, F. R. (2005). On redefining a snap-back repeller. Chaos, Solitons \& Fractals, 25(1), 25-28. 
Niu, W., Shi, J., \& Mou, C. (2016). Analysis of codimension 2 bifurcations for high-dimensional discrete systems using symbolic computation methods. Applied Mathematics and Computation, 273, 934-947.

Sang, B., \& Huang, B. (2017). Bautin bifurcations of a financial system. Electronic Journal of Qualitative Theory of Differential Equations, 2017(95), 1-22.

Wang, D. (2001). Elimination methods. Springer Science \& Business Media.
Wen, G. (2005). Criterion to identify Hopf bifurcations in maps of arbitrary dimension. Physical Review E, 72(2), 026201

Wu, W. T. (2000). Mathematics mechanization: mechanical geometry theorem-proving, mechanical geometry problem-solving, and polynomial equations-solving. Beijing: Science Press.

Yang, L., \& Xia, B. (2005). Real Solution Classification for Parametric Semi-Algebraic Systems. In Algorithmic Algebra and Logic (pp. 281-289) 